\title{
PROPOSTA DE ASSISTÊNCIA DE ENFERMAGEM AO IDOSO COM DEMÊNCIA EM UMA INSTITUIÇÃO DE LONGA PERMANÊNCIA
}

Ananda Spanhol Costa; Universidade Federal de Roraima; anandacosta@outlook.com Raquel Voges Caldart; Universidade Federal de Roraima; raquel.voges@ufrr.br

\section{RESUMO}

Introdução: Idosos com demência apresentam maior risco de institucionalização devido dependência física e mental e à necessidade de cuidados especializados. As instituições de longa permanência para idosos, constituem uma modalidade de moradia, que apesar de suas vantagens, produz isolamento, inatividade física e mental e diminui a qualidade de vida. Objetivo: Elaborar uma proposta de assistência de enfermagem sistematizada para idosos em processo demencial residentes em uma instituição de longa permanência de Roraima. Metodologia: Os dados foram coletados com um instrumento fundamentado na Teoria das Necessidades Humanas Básicas de Wanda de Aguiar Horta e instrumentos de avaliação multidimensional do idoso. Os diagnósticos foram elaborados de acordo com a taxonomia II da North American Nursing Diagnosis Association (2018-2020) e o plano de cuidados, bem como, os resultados esperados foram desenvolvidos com base no Nursing Interventions Classification (2016) e Nursing Outcome Classification (2016), respectivamente. Resultados: Entre os participantes ( $n=23)$, observou-se um elevado percentual (86,9\%) de idosos com déficit cognitivo e todos apresentam graus 1 ou 2 de dependência. Foram elaborados 37 diagnósticos de enfermagem e para elaboração do plano de cuidados, seguiu-se as etapas do processo de enfermagem, agrupando os diagnósticos em seis categorias: cognição e memória; dependência funcional; interações sociais; humor; sono e conforto e; idade, uso de medicamentos e outros problemas de saúde. Conclusão: Este estudo contribui para a divulgação da sistematização da assistência de enfermagem em uma instituição de longa permanência onde se deve proporcionar a manutenção da capacidade funcional, autonomia, independência e qualidade de vida do idoso.

Palavras-chave: Idosos; Sistematização da assistência de enfermagem; Demência; Enfermagem; Instituição de Longa Permanência para Idosos. 